\title{
SEDOPTICA Newsletter
}

\section{Noticias de SEDOPTICA}

\section{Pablo A. Postigo, Lluís F. Marsal, Sara Núñez}

Presidente Anterior del Comité de Nanofotónica de SEDOPTICA, Presidente y Vicepresidenta DOI: dx.doi.org/10.7149/OPA.54.4.xiii

Durante el año 2021 la crisis de la pandemia nos ha seguido generando dificultades pero el ritmo de vacunación parece que este próximo a doblegar la curva de contagios graves. A pesar de ello, el Comité de Nanofotónica ha participado durante 2021 en varios eventos que queremos destacar.

\section{Comité de Nanofotónica en el CEN2021}

Por un lado, los miembros de la Junta Directiva participaron en la nueva edición de la Conferencia Española de Nanofotónica, CEN 2021, que no puedo realizarse en su fecha inicial de 2020 y que tuvo su celebración on-line entre el 20 y 22 de septiembre del año 2021. Esta edición, fue presidida por Isabel Pastoriza-Santos y Sara Núñez-Sánchez (Vicepresidenta actual del Comité de Nanofotónica) del Grupo de Materiales Funcionales del Centro de Investigaciones Biomédicas de la Universidad de Vigo. Durante el evento pudimos disfrutar de un elenco de 10 ponentes plenarios e invitados que tanto desarrollan su investigación en España como en el extranjero.

Ponentes plenarios del CEN2021

Prof. Teri W. Odom, Northwestern University, USA

Prof. Luis M. Liz-Marzán, CIC BiomaGUNE, ES

Dr. Silvia Vignolini, University of Cambridge, UK

Ponentes invitados del CEN2021

Dr. Gabriel Lozano, Instituto de Ciencia de Materiales de Sevilla - ICMS, ES

Dr. Elisabet Romero, Institut Català d'Investigació Química - ICIQ, ES

Dr. Aitzol García Etxarri, Ikerbasque Research Fellow - DIPC, ES

Dr. Irene Fernández Cuesta, University of Hamburg, DE

Dr. Johannes Feist, Universidad Autónoma de Madrid, ES

Prof. Montserrat Calleja, Instituto de Micro y Nanotecnología - IMN, ES

Dr. Pedro David García Fernández, Institut Català de Nanociència i Nanotecnología - ICN2, ES

Para fomentar la participación de miembros de la Sociedad Española de Óptica, se ofreció un descuento del $50 \%$ a todos los socios estudiantes de SEDOPTICA y de la OSA. Así, se registraron un total de 112 participantes. Además, se pudieron disfrutar más de 69 contribuciones incluyendo charlas plenarias, invitadas, orales y Flash talks. La media de visitas diarias a la plataforma fue de 250 visitas con picos de audiencia de más de cincuenta personas asistiendo a una única sesión. Por último, nos gustaría destacar que gracias a los patrocinadores platino (Fotónica21, Portomédica y OSA (ahora OPTICA), pudimos otorgar cinco premios a las mejores presentaciones cortas tipo "Flash Talks".

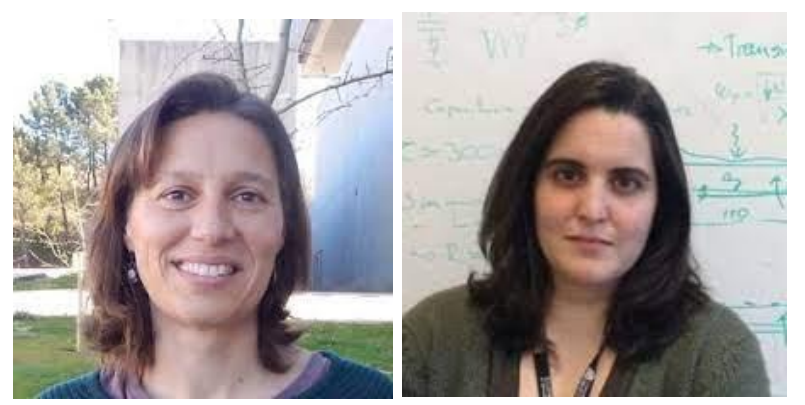

Fig.1. Isabel Pastoriza-Santos y Sara Núñez-Sánchez,, organizadoras del CEN2021. 
Puede verse más información acerca del evento en el siguiente enlace web: http://cen2020.webs.uvigo.es/welcome/

\section{Simposio de Nanofotónica en la RNO2021}

Durante la pasada celebración de la Reunión Nacional de Óptica 2021 (RNO2021) se celebró el primer Simposio del Comité de Nanofotónica, organizado por la Junta Directiva del mismo. Se recibieron mas de 15 trabajos de los cuales 6 se presentaron como orales. Como presentación invitada dentro del Simposio se conto con la charla "Spin-photon interface with a van der Waals heterostructure", impartida por el Prof. Nick Vamivakas del Institute of Optics de la University of Rochester.
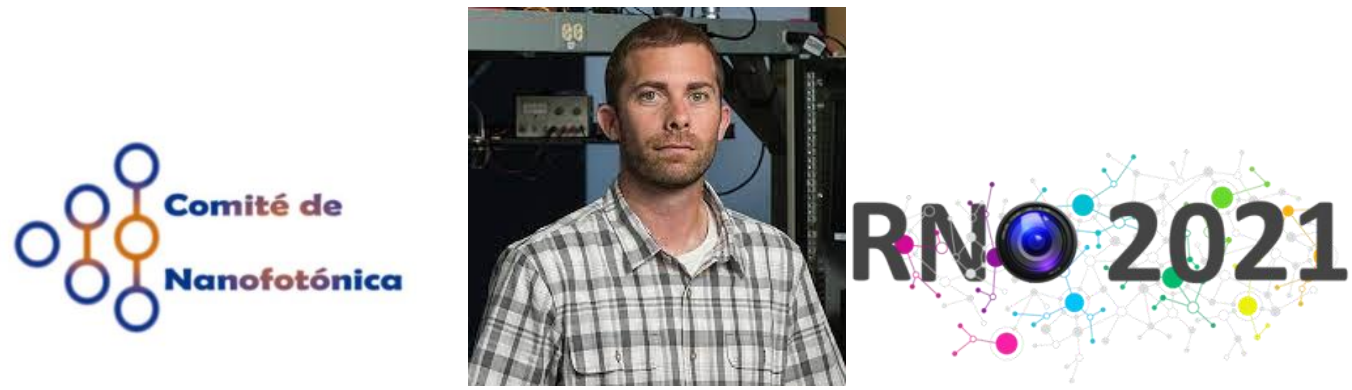

Fig.2. El Prof. Nick Vamivakas ofreció una charla invitada dentro del Simposio de Nanofotónica de la RNO2021

Además, durante dicho Simposio se celebró la 1a Asamblea del Comité de Nanofotónica para la elección de la nueva Junta Directiva del Comité y la aprobación del reglamento. Mediante el acto, que se realizó online, se inició con los agradecimientos del Presidente saliente, Pablo Aitor Postigo, a los esfuerzos del Vicepresidente Lluís F. Marsal y de Sara Núñez, así como del resto de socios, para que el Comité de Nanofotónica se creara dentro de Sedoptica. Tras el agradecimiento se procedió a la elección del nuevo Presidente del Comité, Lluís F. Marsal, y a la nueva Vicepresidenta, Sara Núñez, por unanimidad, y a la aprobación del reglamento del Comité.

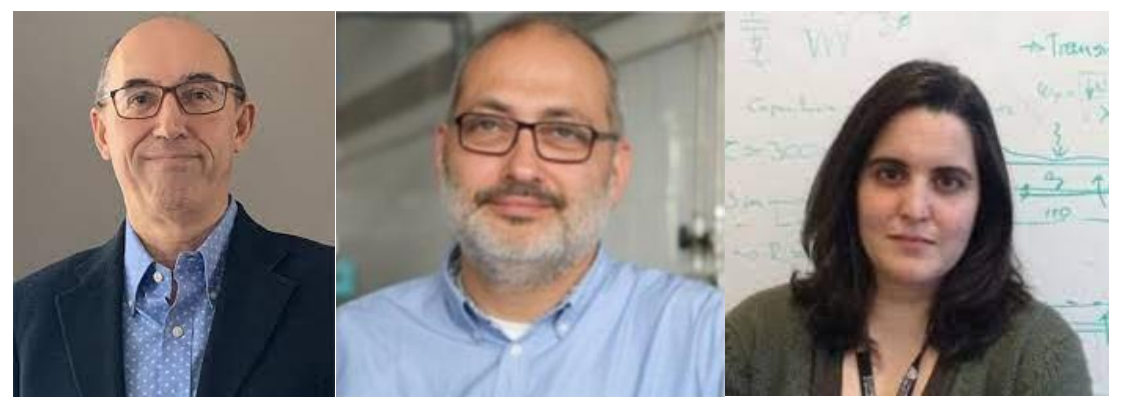

Fig.3. El Prof. P.A. Postigo, Presidente saliente del Comité de Nanofotónica, Lluís F. Marsal (nuevo Presidente y anterior Vicepresidente) y Sara Núñez (nueva Vicepresidenta) elegidos dentro del Simposio de Nanofotónica de la RNO2021

Como nota destacable, uno de los trabajos presentados dentro del Simposio de Nanofotónica fue premiado con el 5o premio de la RNO2021. El titulo del trabajo fue "DISPERSIÓN RAMAN MEJORADA EN SUPERFICIE MEDIADA POR LA ADSORCIÓN DE NANOPARTÍCULAS METÁLICAS GENERADAS CON LÁSER SOBRE ÓXIDO DE GRAFENO" y sus autores fueron Sergio Molina-Prados, A. Cros Stötter, G. Mínguez Vega and N. Garro Martínez, del Institute of New Imaging Technologies (INIT) de la Universitat Jaume I de Castello. 